\title{
Alta frecuencia de sintomatología depresiva en cuidadores de pacientes pediátricos en diálisis peritoneal y hemodiálisis en un hospital público de Lima.
}

High frequency of depressive symptoms in caregivers of pediatric patients on peritoneal dialysis and hemodialysis in a public Hospital in Lima.

Vanessa Lanatta ${ }^{1, a}$, Cristel Quiñones ${ }^{1, a}$, Jose L. Paredes ${ }^{1, a}$, Silvana Sarabia ${ }^{1, b}$, Cristian León ${ }^{1, c}$

\section{RESUMEN}

Objetivo: El objetivo del estudio fue describir la frecuencia de sintomatología depresiva en cuidadores de pacientes pediátricos en diálisis peritoneal y hemodiálisis. Material y Métodos: Estudio descriptivo de corte transversal en el cual participaron 33 cuidadores de pacientes pediátricos con enfermedad renal crónica de la unidad de hemodiálisis y diálisis peritoneal del Hospital Cayetano Heredia en Lima (Perú). El nivel de sintomatología depresiva de los cuidadores fue evaluado mediante el Patient Health Questionnaire (PHQ-9). Resultados: Un total de 54,54\% de cuidadores presentaron sintomatología depresiva; el 9,09\% moderadamente severa, el 18,18\% moderada y el 27,27\% leve; solo el 3,03\% reportó uso de antidepresivos. Todos los cuidadores con depresión moderada y moderadamente severa reportaron algún grado de dificultad para realizar sus labores. Conclusiones: Se evidenció una alta frecuencia de sintomatología depresiva en cuidadores de pacientes pediátricos en diálisis. El reconocimiento de la depresión en los cuidadores principales es importante a fin de conducir intervenciones dirigidas a la preservación de su bienestar emocional.

PALABRAS CLAVE: Depresión, diálisis, cuidador, pediatría.

\section{SUMMARY}

Objective: To describe the frequency of depressive symptomatology in caregivers of pediatric patients on peritoneal dialysis and hemodialysis. Material and methods: Descriptive, cross-sectional study of 33 caregivers of pediatric patients with Chronic Kidney Disease, under treatment in the Hemodialysis and Peritoneal Dialysis Unit of the Cayetano Heredia Hospital in Lima (Peru). The participants completed the Patient Health Questionnaire (PHQ9), which assessed their degree of depressive symptomatology. Results: A total of 54.54\% of caregivers showed depressive symptomatology; $9.09 \%$ moderately severe, $18.18 \%$ moderate and $27.27 \%$ mild; only $3.03 \%$ reported intake of antidepressants. All caregivers with moderate and moderately severe depression reported some degree of difficulty in performing their tasks. Conclusions: A high frequency of depressive symptomatology in caregivers of pediatric patients on dialysis was found. The recognition of depression in primary caregivers is important, in order to carry out interventions aimed at maintaining their emotional well-being.

KEYWORDS: Depression, dialysis, caregivers, pediatrics.

Facultad de Medicina, Universidad Peruana Cayetano Heredia, Lima, Perú.

a Médico; ${ }^{b}$ Médico especialista en psiquiatría; ${ }^{\mathrm{c}}$ Médico especialista en nefrología. 
Alta frecuencia de sintomatología depresiva en cuidadores de pacientes pediátricos en diálisis peritoneal.

\section{INTRODUCCIÓN}

La enfermedad renal crónica terminal (ERC) es la pérdida progresiva e irreversible de la función renal que lleva al paciente a la necesidad de terapia de sustitución renal o al trasplante renal (1). Según la Organización Mundial de la Salud (OMS), la ERC afecta a cerca del $10 \%$ de la población mundial, por lo que es considerada un problema de salud pública a nivel mundial (2). En el Perú, un estudio en el 2015 en Lima y Tumbes encontró una prevalencia de ERC de $16,8 \%(3)$.

Los tratamientos de sustitución renal son altamente invasivos y demandantes, e implican un alto costo físico, psicosocial y económico para el paciente y su entorno; entre ellos se encuentran el trasplante renal, la hemodiálisis y la diálisis peritoneal. En la hemodiálisis, una máquina filtradora de sangre extrae los desechos urémicos del paciente, para lo que debe asistir tres veces por semana al centro asistencial y permanecer de 3 a 4 horas (4). La diálisis peritoneal es un proceso por el cual el líquido de diálisis es instilado al espacio peritoneal; el líquido es posteriormente extraído, llevándose consigo las toxinas (5).

$\mathrm{La}$ incertidumbre respecto al futuro, las restricciones dietéticas, la dependencia a la máquina de diálisis, los cambios en las relaciones familiares, sociales y en el aspecto físico, explican la aparición de diferentes trastornos emocionales en los pacientes con esta condición, siendo la depresión el desorden psiquiátrico más común $(6,7)$. La ERC se considera una enfermedad severa que provoca sufrimiento tanto en el paciente como en la familia, por el alto riesgo de complicaciones médicas y la repercusión que tiene en la economía familiar. Además, los pacientes en diálisis experimentan una serie de síntomas somáticos y tienen una menor participación en actividades sociales, ocupacionales y recreacionales (8). La combinación de los síntomas físicos y psicológicos resulta en una reducción significativa de la calidad de vida, contribuyendo de esta manera al desarrollo de la depresión (9).

En pacientes pediátricos con ERC, la progresión normal del niño hacia la independencia es impedida, y las preocupaciones sobre el desarrollo se incrementan a medida que se observa un retraso en su crecimiento. Además, la perspectiva de toda una vida con terapia de reemplazo renal y la posibilidad de complicaciones y/o la muerte les dificulta lograr una infancia o adolescencia normal (10). Un estudio realizado en el Hospital Cayetano Heredia $(\mathrm{HCH})$ encontró que $10,45 \%$ de pacientes pediátricos en diálisis presentaba sintomatología depresiva elevada, y $43,48 \%$ sintomatología depresiva leve (11).

Se sabe que las enfermedades crónicas están asociadas a depresión en los pacientes y en sus familias. En el caso de los cuidadores de pacientes pediátricos con ERC terminal, la carga aumenta significativamente a medida que las deficiencias funcionales y cognitivas limitan la capacidad del paciente para cuidarse a sí mismo. Asimismo los cuidadores deben soportar la carga psicológica y emocional de tener un niño con una enfermedad crónica (12).

En el plano familiar, el estilo de vida se centra en el enfermo, pues su cuidador en muchos casos prioriza el cuidado y bienestar del paciente y deja sus otras obligaciones de lado (13); un estudio en Taiwán evidenció que la prevalencia de depresión en cuidadores de niños con ERC en diálisis es mayor comparado con cuidadores de niños sanos (14).

En Pakistán, un estudio realizado en la unidad de hemodiálisis de un hospital comparó la frecuencia de depresión en los pacientes adultos con ERC terminal y en sus cuidadores, evidenciándose depresión moderada a severa en $75 \%$ de pacientes y $60 \%$ de sus cuidadores (15). A pesar de esto, poco se sabe sobre la frecuencia de sintomatología depresiva de los cuidadores de pacientes pediátricos en diálisis en nuestro país.

El objetivo de este estudio fue describir la frecuencia de sintomatología depresiva en los cuidadores de los pacientes pediátricos en diálisis peritoneal y hemodiálisis del $\mathrm{HCH}$, y las características sociodemográficas del cuidador y del paciente.

\section{MATERIAL Y MÉTODOS}

El presente es un estudio de tipo observacional - transversal. Se incluyeron a todos los cuidadores de pacientes pediátricos en diálisis peritoneal y hemodiálisis del $\mathrm{HCH}$. La unidad de diálisis de dicho hospital cuenta con 15 pacientes pediátricos en hemodiálisis y 20 en diálisis peritoneal.

Los criterios de inclusión fueron: edad mayor o igual a 18 años, ser reconocido como cuidador principal del paciente, que éste haya estado en tratamiento de diálisis crónica por un periodo igual o mayor a 3 meses, y aceptar participar en el estudio mediante la firma del consentimiento informado. Se 
excluyeron a participantes que llenaron menos del $10 \%$ de la encuesta.

Cuidador principal fue definido como: "persona del entorno del paciente que asume voluntariamente el papel de responsable del mismo, en un amplio sentido; dispuesta a tomar decisiones por el paciente y a cubrir necesidades básicas del mismo"(16).

Se utilizó un cuestionario anónimo de 17 preguntas compuesto de dos partes. En la primera parte, los datos sociodemográficos obtenidos fueron: edad, sexo, procedencia, estado civil, nivel socio-económico (categorizado según el ingreso familiar mensual) y uso de fármacos neuropsiquiátricos. La segunda parte fue el cuestionario Patient Health Questionnaire (PHQ-9) (17), cuestionario autoadministrado y validado en el Perú por la Dirección de Salud Mental del Ministerio de Salud (MINSA) y el Instituto Nacional de Salud (INS) (16). El PHQ-9 consta de 10 preguntas, 9 relacionadas directamente con sintomatología depresiva durante las dos semanas previas, y una con la dificultad en el trabajo, labores domésticas y relaciones interpersonales asociadas con los síntomas.

Los resultados del PHQ-9 fueron clasificados como: síntomas mínimos o ausencia de depresión (de 0-4), depresión leve (de 5-9), depresión moderada (de 10-14), depresión moderadamente severa (de 15-19) y depresión severa (de 20-27) (17). La variable "dificultad en el trabajo, labores domésticas y relaciones interpersonales a consecuencia de la depresión" fue categorizada en: No presentó dificultad ("Nada difícil"), dificultad leve ("Un poco difícil"), dificultad moderada ("Muy difícil") y dificultad severa (“Extremadamente difícil").

Con respecto a las características clínicas del paciente, se consideraron el número de hospitalizaciones en el último año por complicaciones relacionadas a la terapia o condición de fondo, y el tiempo en diálisis en meses según la historia clínica de los pacientes.

Los datos sociodemográficos y de sintomatología depresiva fueron descritos según frecuencias $y$ porcentajes para variables cualitativas, y medias y desviaciones estándar para variables cuantitativas.

Se contó con la revisión y aprobación del comité de ética de la Universidad Peruana Cayetano Heredia y del Comité Institucional de Ética en la Investigación del $\mathrm{HCH}$. Se registró el nombre del paciente en diálisis y se tomaron los datos de la historia clínica. Los datos fueron codificados en una base de datos con único acceso a los investigadores y sin posibilidad de identificar a los participantes. Se proporcionó una hoja informativa sobre los síntomas de depresión, como prevenirla y donde buscar ayuda a todos los participantes.

\section{RESULTADOS}

\section{Factores sociodemográficos}

Se invitó a participar a 35 cuidadores, de los cuales 33 (94,28\%) aceptaron y firmaron el consentimiento informado. La tabla 1 proporciona las características sociodemográficas y uso de fármacos neuropsiquiátricos de los cuidadores enrolados. El $84,84 \%$ tenían entre 30 y 59 , años y la mediana de edad fue 39 años $(R I Q=9,52)$. La mayoría $(69,7 \% \mathrm{n}=23)$ de cuidadores fueron del sexo femenino, procedentes de Lima $(81,81 \% n=27)$, y convivientes $(42,42 \% n=14)$; y el $90,9 \%(n=30)$ de cuidadores pertenecían al nivel socio económico E. Un cuidador (3,03\%) reportó tomar antidepresivos.

\section{Frecuencia de depresión y dificultad percibida en el trabajo, labores domésticas y relaciones interpersonales}

Los resultados del cuestionario PHQ-9 están presentados en la tabla 2. Ningún cuidador presentó depresión severa; el 9,09\% $(\mathrm{n}=3)$ presentaron depresión moderadamente severa; el 18,18\% ( $\mathrm{n}=6)$ depresión moderada; el 27,27\% ( $\mathrm{n}=9)$ depresión leve, y el $45,45 \%(n=15)$ depresión mínima o ausente. El $45,45 \%(n=15)$ refirieron dificultad leve en el trabajo, labores domésticas y en sus relaciones interpersonales como consecuencia de los síntomas depresivos, el $21,21 \%(\mathrm{n}=7)$ dificultad moderada y solo el 3,03\% $(\mathrm{n}=1)$ dificultad severa.

La tabla 3 proporciona la relación entre el cuestionario PHQ-9 y la dificultad en el trabajo, labores domésticas y relaciones interpersonales de los participantes De los participantes con depresión moderada, el 33,33\% $(\mathrm{n}=2)$ refirieron poca dificultad, y el $66,67 \%(n=4)$ mucha dificultad. Por último, de los pacientes que presentaron depresión moderadamente severa, el $66,67 \%(\mathrm{n}=2)$ respondieron que tenían mucha dificultad, y el 33,37\% extrema dificultad.

La tabla 4 contiene las características clínicas de los pacientes pediátricos en diálisis; más de la mitad 
Alta frecuencia de sintomatología depresiva en cuidadores de pacientes pediátricos en diálisis peritoneal.

Tabla 1. Características sociodemográficas y uso de fármacos neuropsiquiátricos de cuidadores de pacientes pediátricos en hemodiálisis y diálisis peritoneal en un hospital público en Lima, Perú.

\begin{tabular}{|c|c|c|c|}
\hline & Variable & $\mathbf{n}$ & Porcentaje (\%) \\
\hline & $20-29$ & 4 & 12,12 \\
\hline \multirow[t]{2}{*}{ Edad } & $30-59$ & 28 & 84,84 \\
\hline & $60-69$ & 1 & 3,03 \\
\hline \multirow{2}{*}{ Sexo } & Femenino & 23 & 69,70 \\
\hline & Masculino & 10 & 30,30 \\
\hline \multirow{3}{*}{ Procedencia } & Lima & 27 & 81,81 \\
\hline & Provincia & 6 & 18,18 \\
\hline & Soltero(a) & 8 & 24,24 \\
\hline \multirow{3}{*}{ Estado civil } & Casado(a) & 11 & 33,33 \\
\hline & Conviviente & 14 & 42,42 \\
\hline & Viudo(a) & 0 & 0 \\
\hline \multirow{5}{*}{$\begin{array}{l}\text { Nivel socio económico } \\
\text { (NSE) }\end{array}$} & NSE-A & 0 & 0 \\
\hline & NSE-B & 0 & 0 \\
\hline & NSE-C & 1 & 3,03 \\
\hline & NSE-D & 2 & 6,06 \\
\hline & NSE-E & 30 & 90,9 \\
\hline \multirow{2}{*}{$\begin{array}{l}\text { Uso de fármacos } \\
\text { neuropsiquiátricos }\end{array}$} & Sí (Antidepresivo) & 1 & 3,03 \\
\hline & No & 32 & 96,97 \\
\hline
\end{tabular}

de los pacientes $(51,51 \%, \mathrm{n}=17)$ fueron hospitalizados de 1 a 2 veces en el último año. El 33,33\% (n=12) de los pacientes llevan menos de 2 años en terapia de reemplazo renal, el 30,3\% $(n=10)$ entre 2 y 4 años, y el 36,3\% ( $\mathrm{n}=12)$ más de 4 años. El tiempo en diálisis mínimo fue de 3 meses y el tiempo máximo de 110 meses.

\section{DISCUSIÓN}

El presente trabajo de investigación reveló que un $54,54 \%$ de cuidadores de pacientes pediátricos en diálisis presentaron algún grado de depresión, resultados que muestran concordancia con un estudio que enroló a 180 cuidadores de pacientes en 
Tabla 2. Frecuencia de sintomatología depresiva según el cuestionario PHQ-9 de cuidadores de pacientes pediátricos en hemodiálisis y diálisis peritoneal en un hospital público en Lima, Perú.

\begin{tabular}{|c|c|c|c|}
\hline & Score PHQ-9 & $\mathrm{n}$ & Porcentaje (\%) \\
\hline \multirow{5}{*}{ Depresión } & Mínima o ausente & 15 & 45,45 \\
\hline & Leve & 9 & 27,27 \\
\hline & Moderada & 6 & 18,18 \\
\hline & Moderadamente severa & 3 & 9,09 \\
\hline & Severa & 0 & 0 \\
\hline \multirow{4}{*}{$\begin{array}{l}\text { Dificultad } \\
\text { percibida }\end{array}$} & Nada difícil & 10 & 30,3 \\
\hline & Un poco difícil & 15 & 45,45 \\
\hline & Muy difícil & 7 & 21,21 \\
\hline & Extremadamente difícil & 1 & 3,03 \\
\hline
\end{tabular}

Tabla 3. Relación entre la sintomatología depresiva según el cuestionario PHQ-9 y dificultad en el trabajo, labores domésticas y relaciones interpersonales percibida por cuidadores de pacientes pediátricos en hemodiálisis y diálisis peritoneal en un hospital público en Lima, Perú.

\begin{tabular}{lcccc}
\hline PHQ-9 & Nada difícil & Un poco difícil & Muy difícil & Extremadamente difícil \\
\hline Mínima o ausente & $55,33 \%(\mathrm{n}=8)$ & $46,67 \%(\mathrm{n}=7)$ & $0 \%(\mathrm{n}=0)$ & $0 \%(\mathrm{n}=0)$ \\
Leve & $22,22 \%(\mathrm{n}=2)$ & $66,67 \%(\mathrm{n}=6)$ & $11,11 \%(\mathrm{n}=1)$ & $0 \%(\mathrm{n}=0)$ \\
Moderada & $0 \%(\mathrm{n}=0)$ & $33,33 \%(\mathrm{n}=2)$ & $66,67 \%(\mathrm{n}=4)$ & $0 \%(\mathrm{n}=0)$ \\
Moderadamente severa & $0 \%(\mathrm{n}=0)$ & $0 \%(\mathrm{n}=0)$ & $66,67 \%(\mathrm{n}=2)$ & $33,33 \%(\mathrm{n}=1)$ \\
Severa & $0 \%(\mathrm{n}=0)$ & $0 \%(\mathrm{n}=0)$ & $0 \%(\mathrm{n}=0)$ & $0 \%(\mathrm{n}=0)$ \\
\hline
\end{tabular}

Tabla 4. Características clínicas de pacientes pediátricos en hemodiálisis y diálisis peritoneal en un hospital público en Lima, Perú.

\begin{tabular}{lcc}
\hline \multicolumn{1}{c}{ Características clínicas } & $\mathbf{n}$ & Porcentaje (\%) \\
\hline Número de hospitalizaciones & & \\
$\mathbf{0}$ & 13 & 39,39 \\
$\mathbf{1 - 2}$ & 17 & 51,51 \\
$\mathbf{3 - 4}$ & 2 & 6,06 \\
$\mathbf{5 - 6}$ & 0 & 0 \\
$\mathbf{7 - 8}$ & 1 & 3,03 \\
Tiempo en diálisis & & \\
$\quad$ Menos de 2 años & 11 & 33,33 \\
$\quad$ Entre 2 y 4 años & 10 & 30,30 \\
$\quad$ Mas de 4 años & 12 & 36,36 \\
\hline
\end{tabular}

hemodiálisis en Pakistán, en el cual un 65,1\% de ellos presentaron algún grado de depresión; este estudio utilizó el Inventario de Depresión de Beck, el cual tiene una mayor sensibilidad (93\%) que el PHQ-9 (15), lo cual podría explicar la diferencia en los resultados.

Un estudio realizado en Etiopía para evaluar la confiabilidad y validez del PHQ-9 como cribado para diagnosticar trastorno depresivo mayor en adultos, demostró que un punto de corte de 10 ofrecía un poder discriminatorio óptimo para dicho diagnóstico, teniendo una sensibilidad de $86 \%$ y una especificidad del 67\% (18); en base a esto, el 27,27\% de los cuidadores de nuestro estudio presentaron un probable trastorno depresivo mayor.

La frecuencia de depresión en nuestro estudio es considerablemente mayor a la de la población general; por ejemplo, el Estudio Epidemiológico Metropolitano 
Alta frecuencia de sintomatología depresiva en cuidadores de pacientes pediátricos en diálisis peritoneal.

de Lima y Callao realizado en el 2012 en 5340 hogares, encontró una frecuencia de depresión de $17,3 \%$. En nuestro estudio, solo un participante $(3,03 \%)$ refirió tomar antidepresivos; es importante reconocer que no existen programas de soporte ni detección de depresión para cuidadores en el $\mathrm{HCH}$.

Factores asociados a la depresión en cuidadores de pacientes pediátricos en diálisis previamente reconocidos son ser mujer, pertenecer a un estrato socioeconómico bajo y que el paciente esté un tiempo mayor a 4 años en diálisis $(14,16)$.

Debido al tamaño muestral, en nuestro estudio no se pudieron realizar asociaciones estadísticas, sin embargo, es importante reconocer que la mayoría de los participantes fueron mujeres, pertenecían a un estrato socioeconómico bajo y el $36,3 \%$ de los pacientes llevaban más de 4 años en terapia de remplazo renal. Otras limitaciones fueron que, al ser una encuesta autoadministrada puede haber existido un sesgo de omisión de sintomatología depresiva en los participantes. Es importante diseñar estudios en poblaciones más grandes, en otros centros de diálisis tanto públicos como privados, para poder representar mejor la realidad de nuestro medio e implementar intervenciones pertinentes en los cuidadores.

En conclusión, este estudio evidencia una alta frecuencia de sintomatología depresiva en cuidadores de pacientes pediátricos en diálisis del $\mathrm{HCH}$. Es necesario crear programas de salud mental que mejoren la calidad de vida de los mismos.

Agradecimientos: A la unidad de diálisis del $\mathrm{HCH}$ y a todos los participantes del estudio.

Fuentes de financiamiento: Autofinanciado.

Conflictos de interés: Los autores no reportan ningún conflicto de interés.

\section{REFERENCIAS BIBLIOGRÁFICAS}

1. Bargman J, Skorecki G. Nefropatía crónica y uremia. En: Kasper D, Hauser S, Jameson J, Fauci A, Longo D, Loscalzo J, et al, editors. Harrison's Principles of Internal Medicine. $19^{\text {th }}$ Edition. New York: Mc Graw Hill; 2015. p. 1761-1772.

2. Organización Panamericana de la Salud. La OPS/ OMS y la Sociedad Latinoamericana de Nefrología llaman a prevenir la enfermedad renal y a mejorar el acceso al tratamiento. Washington
DC: Organización Panamericana de la Salud; 2015. (Citado el 10 de enero del 2019) Disponible en:https://www.paho.org/hq/index.php?option=com content\&view=article\&id=10542:2015-opsomssociedad-latinoamericana-nefrologia-enfermedadrenal-mejorar-tratamiento\&Itemid $=1926 \&$ lang $=\mathrm{pt}$

3. Francis ER, Kuo CC, Bernabe-Ortiz A, Nessel L, Gilman RH, Checkley W, et al. Burden of chronic kidney disease in resource-limited settings from Peru: a population-based study. BMC Nephrol. 2015; 16:114.

4. Kidney International Supplements. Chapter 3: Management of progression and complications of CKD. Kidney Int Suppl (2011). 2013; 3(1): 73-90. doi: 10.1038/kisup.2012.66

5. Piaskowski P. Hemodiálisis y diálisis peritoneal. En: Friedman C, Newson W, editores. Conceptos básicos de control de infecciones de IFIC. 2da Edición. Irlanda: International Federation of Infection Control; 2011. p. 289-302.

6. Rodríguez E, Campillo M, Avilés M,. Calidad de vida en pacientes con insuficiencia renal crónica y su familia. Hacer Psicologia. 2013;6:0-0. (Citado 26 de enero de 2019) Disponible en: https://www.uv.mx/ psicologia/files/2013/06/Calidad-de-vida.pdf

7. Kimmel PL, Cukor D, Cohen SD, Peterson RA. Depression in end-stage renal disease patients: a critical review. Adv Chronic Kidney Dis. 2007; 14(4): 328-34.

8. Purnell TS, Auguste P, Crews DC, LampreaMontealegre J, Olufade T, Greer R et al. Comparison of life participation activities among adults treated by hemodialysis, peritoneal dialysis, and kidney transplantation: a systematic review. Am J Kidney Dis. 2013; 62(5): 953-73.

9. Kimmel PL, Patel SS. Quality of life in patients with chronic kidney disease: focus on end-stage renal disease treated with hemodialysis. Semin Nephrol. 2006; 26(1):68-79.

10. Srivastava T, Warady B. Overview of the managament of chronic kidney disease in children. UpToDate; 2016. (Citado 26 de enero de 2019) Disponible en: https://www.uptodate.com/contents/overview-of-themanagement-of-chronic-kidney-disease-in-children

11. Hernandez EG, Loza R, Vargas H, Jara MF. Depressive symptomatology in children and adolescents with chronic renal insufficiency undergoing chronic dialysis. Int $\mathrm{J}$ Nephrol. 2011;2011:798692.

12. Gayomali C, Sutherland S, Finkelstein F. The challenge for the caregiver of the patient with chronic kidney disease. Nephrol Dial Transplant. 2008; 23 (12): 3749-3751.

13. Schneider RA. Fatigue among caregivers of chronic renal failure patients: a principal components analysis. Nephrol Nurs J. 2003; 30(6):629-33. 
14. Tsai TC, Liu SI, Tsai JD, Chou LH. Psychosocial effects on caregivers for children on chronic peritoneal dialysis. Kidney International. 2006; 70(11): 19831987.

15. Saeed Z, Ahmad A, Shakoor A, Ghafoor F, Kanwal S. Depression in patients on hemodialysis and their caregivers. Saudi J Kidney Dis Transpl. 2012; 23(5): 946-52.

16. Saavedra J, Schaeffer V, Vargas H, Robles Y, Pomalima R, González S, et al. Estudio epidemiológico de salud mental en Lima metropolitana y Callao-Replicación 2012. Perú: Anales de Salud mental. 2012; 29(S1): 0-0. (Citado 26 de enero de 2019) Disponible en: http://www. insm.gob.pe/investigacion/archivos/ estudios/2012\%20ASM\%20-EESM\%20-LM.pdf
17. Grey L. Caregiver depression: A growing mental health concern. San Francisco: Family Caregiver Alliance; 2003. (Citado 26 de enero de 2019) Disponible en: https:/pdfs.semanticscholar.org/a7e6/ 792ffc42e8ba9b9805e76ceeedf3abc2f77a.pdf

18. Guerrero J, Romero G, Martinez M, Martinez R, Rosas S. Percepción del cuidador primario sobre las intervenciones de enfermería al paciente en estado terminal. Rev Enferm Inst Mex Seguro Soc. 2016; 24(2):91-8.

Recibido: 09/07/2019 Aceptado: 06/09/2019 